\title{
ORIGINAL ARTICLE \\ Adapted sport effect on postural control after spinal cord injury
}

\author{
PE Magnani ${ }^{1}$, NR Marques ${ }^{2}$, AC Junior ${ }^{3,4}$ and DCC de Abreu ${ }^{1}$
}

\begin{abstract}
Study design: Cross-sectional study.
Objective: The aim of this study was to compare trunk muscle activation during anterior and lateral reach in athletic and sedentary individuals with spinal cord injury (SCI) and able-bodied people.

Settings: University Hospital-UNICAMP, Campinas, Brazil.

Methods: Individuals with complete traumatic $\mathrm{SCl}$ and thoracic neurological level were separated into two groups: sedentary (SSCI: $n=10$ ) and physically active (PASCI: $n=10$ ). The control group (C: $n=10$ ) without SCI was assessed. Trunk muscle activation was recorded during reach and grasp tasks. The significant level was set at $P<0.05$.

Results: The control group showed a highest mean activation for left longissimus muscle during all activities $(P<0.05)$. The PASCI group presented significant highest activation for left iliocostalis muscles during all activities, except in the anterior reach task of $90 \%$ maximum reach (anterior reach (AR) 75: $P=0.02$; right lateral reach (RLR) 75: $P=0.03$; RLR90: $P=0.01$ ). The SSCI group presented highest activation for the left iliocostalis during the right lateral reach task of 75 and $90 \%$ maximum reach and right iliocostalis during the anterior reach task of $75 \%$ maximum reach (AR75: $P=0.007$; RLR75: $P=0.02 ; \mathrm{RLR} 90: P=0.03$ ). A different pattern of muscle activation between the control group and the groups with $\mathrm{SCl}$ was observed.

Conclusion: Our results indicated that sports practice did not affect the trunk muscle activation in people with paraplegia. However, the pattern muscle activation in individuals with $\mathrm{SCl}$ is different compared with people without $\mathrm{SCl}$ during anterior reach tasks.
\end{abstract}

Spinal Cord (2016) 54, 1188-1196; doi:10.1038/sc.2016.73; published online 31 May 2016

\section{INTRODUCTION}

Postural control, the ability to maintain, achieve or restore a state of balance during any posture and activity, ${ }^{1,2}$ is essential to achieve independence in daily activities.

Musculoskeletal and neural structures are required to maintain postural control during static and dynamic movements. These structures are composed of muscles, joints and ligaments, the sensory perception process, and the superior level process, which are necessary to detect stimuli, process the information and plan the motor act. ${ }^{3}$

Sensory information is sent to the central nervous system, which is necessary for promoting a motor response for postural corrections in unstable situations. ${ }^{1}$ The maintenance of stability is a dynamic process, requiring equilibrium between stabilization and destabilization forces. ${ }^{3}$ Thus, the sensory input must be integrated and converted to a motor output, which is conducted outside the spine by the motor pathways. However, after a spinal cord injury (SCI), the afferent and efferent pathways are damaged, resulting in postural control changes. ${ }^{4,5}$

According to previous studies, people with SCI have less stability in sitting posture compared with people without SCI. Moreover, the higher the injury, the more difficult it is to maintain postural control and more compensation while sitting. ${ }^{6,7}$
Previous studies also demonstrated that people with SCI had an abnormal biomechanic (kinematic and electromyographic (EMG)) pattern while performing trunk movements such as flexion, extension and lateral bending. According to Seelen et al. ${ }^{8}$ and Chen et al. ${ }^{9}$ people with SCI had increased postural sway as the height of the injury increased. In addition, Seelen et al. ${ }^{8}$ identified that a different muscle activation pattern may occur in order to compensate the functional deficit and trunk instability due to SCI dysfunction.

According to Refet and Hasan, ${ }^{10}$ individuals with SCI and poor control of spinal stabilization muscles performed functional reach activities slower, had increased energy expenditure and were unable to keep the trunk straight while sitting.

In order to maintain postural control, the body uses compensatory and anticipatory strategies. The compensatory system quickly corrects postural imbalances through synergy muscles, and the anticipatory system provides postural adjustments prior to voluntary movements to minimize postural disturbances. ${ }^{11}$ Because of the specific situation there is a need for constant adjustment between perception and action strategies that allow the detection and correction of minor motor errors, essential for the desired task to occur properly. ${ }^{12}$ When errors are not resolved, the motor performance will not be adequate,

${ }^{1}$ Physiotherapy Course, Department of Biomechanics, Medicine and Rehabilitation of Locomotor System, School of Medicine, University of São Paulo (USP), Ribeirão Preto, SP, Brazil; ${ }^{2}$ Physiotherapy Course, Department of Physical Therapy and Occupational Therapy, Faculty of Philosophy and Science, São Paulo State University, Marilia, SP, Brazil; ${ }^{3}$ Biomechanics and Rehabilitation Lab, Department of Orthopedics and Traumatology, Faculty of Medical Sciences, University of Campinas-Unicamp, Campinas, SP, Brazil and ${ }^{4}$ Biocybernetics and Rehabilitation Engineering Lab, Department of Electrical Engineering, University of São Paulo, São Carlos, Brazil

Correspondence: Professor DCC de Abreu, Physiotherapy Course, Department of Biomechanics, Medicine and Rehabilitation of Locomotor System, School of Medicine, University of São Paulo (USP), Avenida Bandeirantes, 3900; Ribeirão Preto, SP 14049-900, Brazil.

E-mail: dabreu@fmrp.usp.br

Received 28 April 2015; revised 1 April 2016; accepted 6 April 2016; published online 31 May 2016 
resulting in undesirable positions or falls, which justifies the difficulty for individuals with SCI to maintain postural control.

Previous studies showed several benefits of adapted sports in people with SCI, such as improvement of functionality; increased oxygen uptake; reduced risk of cardiovascular diseases, respiratory infections, incidence of medical complications and hospitalizations; increased life expectancy; improvement of agility; ${ }^{13}$ and motor skills and quality of life. ${ }^{14}$ However, the effect of adapted sports on muscle activation, postural control, performance of activities of daily living and functional independence in people with SCI remains unclear.

Surface electromyography is a reliable method to assess muscle activation and explore the physiological processes that occur in muscle force generation during a body movement. ${ }^{15,16}$ With respect to this, the aim of the study was to compare the activated trunk muscles during anterior and lateral reach tasks in athletes and sedentary individuals with thoracic SCI.

Our hypothesis is that adapted sports can alter trunk muscle activation in flexion and lateral inclination during reach and grasp movements.

\section{MATERIALS AND METHODS}

A cross-sectional study was used. This study involved men $(n=20)$ between 20 and 60 years of age with at least 12 months of complete SCI and neurological impairment level between T3 and T10 according to the American Spinal Injury Association scale. The control group (C) included 10 able-bodied men within the same age range as the individuals with paraplegia.

The exclusion criteria were history of cranial-encephalic trauma associated with SCI, the presence of cardiorespiratory diseases and orthopedic disorders, vestibulopathies, depression, cognitive deficits or psychiatric diseases, and diabetes mellitus. None of the participants reported shoulder pain or limited range of motion in the upper limbs.

Participants were separated into two groups: sedentary with SCI (SSCI, $n=10)$ and physically active with SCI (PASCI, $n=10$ ).

Physically active individuals participated in creational or competitive sport or physical activity for at least $1 \mathrm{~h}$ for three or more times per week for at least 6 months. Individuals with SCI played basketball (20\%), handball $(60 \%)$, badminton (10\%) and tennis (10\%).
The sedentary group included individuals with SCI who did not play an adapted sport and only performed activities of daily living such as wheelchair propulsion, house cleaning and others.

Participants were recruited in the Laboratory of Biomechanics and Rehabilitation of the Locomotor System of UNICAMP Hospital and the University of Physical Education of UNICAMP. The American Spinal Injury Association protocol to determine the level of injury was performed by an experienced professional ${ }^{17}$ before the functional reach test evaluation.

The SCI levels were between T3 and T10. The SSCI group was composed of individuals with the following level of injury: one with T4, four with T5, one with T6, two with T7, one with T8 and one with T9. The PASCI group was composed of individuals with the following level of injury: one with T3, one with T4, two with T6, one with T7, two with T8, two with T9 and one with T10.

All participants signed an informed consent, and the study was approved by the local ethics committee (Process Identification Number: 12515/2013). In addition, we certify that all applicable institutional and governmental regulations concerning the ethical use of human volunteers were followed during the course of this research.

\section{Clinical evaluations}

Clinical data on age, time of injury (for individuals with SCI) and practice times were recorded. In addition, weight and height were assessed.

\section{Quantitative evaluation}

The trunk flexion evaluation was performed under different conditions: forward and lateral reach tasks. The sitting functional reach ${ }^{18}$ is a clinical evaluation of postural control by measuring an individual's maximum reach. This test is a reliable assessment and can be used on individuals with SCI. ${ }^{19}$

Before the evaluation, familiarization of two repetitions of each movement with $3 \mathrm{~min}$ of rest between trials was conducted.

For the maximum forward reach, the participant was seated in a wheelchair without upper extremity support and laterally positioned against the wall with both shoulders flexed at $90^{\circ}$. A measuring tape was parallel to the floor, positioned at the height of the acromion. The participant was instructed to lean forward as far as possible without losing balance or moving the wheelchair. The displacement was measured by the tape. The subject performed three forward reach trials, and the mean of the three trials was recorded for data analysis.

For maximum lateral reach, the participant was instructed to stretch his/her arms alongside the chair as close to the body as possible, and the distance was

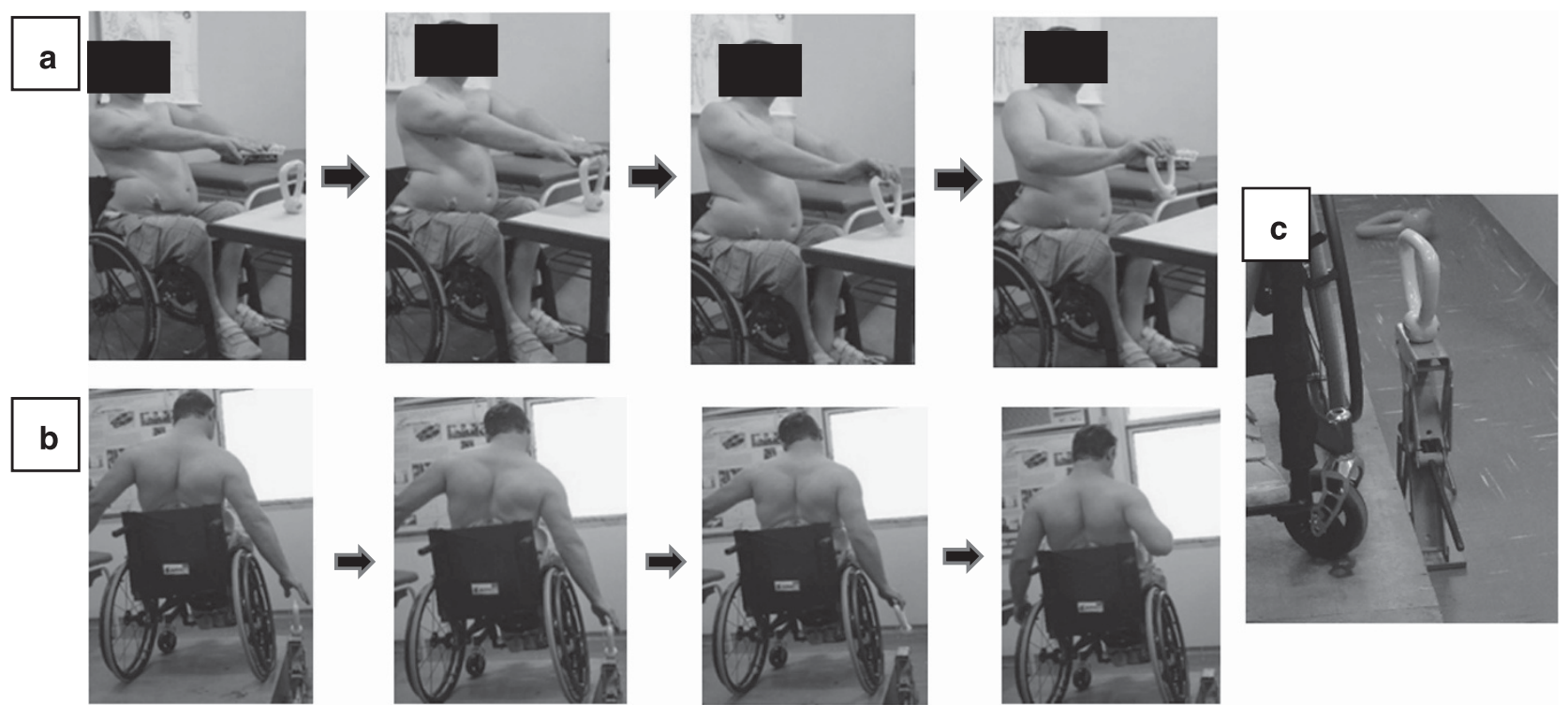

Figure 1 (a) Motion sequence in the anterior reach and the palmar grasp test. (b) Motion sequence in the right lateral reach test. (c) Wheelchair on top of a 19- $\mathrm{cm}$ wooden platform. Height adjustments were made with a mechanical jack. 
measured from the metacarpophalangeal of the second finger to the ground. The subject performed three lateral reach trials, and the mean of the three trials was recorded for data analysis. During lateral trunk flexion, the participant could not grab the wheelchair because the contralateral elbow was extended, and the arm was parallel to the trunk.

The measurements were used to set reach and grasp test parameters, which were 75 and $90 \%$ of the maximum anterior and lateral reach.

For anterior reach and grasp, an adjustable table was placed in front of the subjects with a height tailored to their xiphoid process. An object was placed on the table at 75\% (AR75) and 90\% (AR90) of maximum capacity. The participants had to bring the object with both hands to their laps (Figure 1).

The right lateral reach and grasp test were performed with the dominant hand. The object was placed alongside the wheelchair at 75\% (RLR75) and 90\% (RLR90) of maximum lateral reach. The test consisted of bringing the object with one hand to the participant's lap. The subjects could not use their opposite hand to hold the chair or aid in the task.

During this test, so we can place the object in the previously stipulated distance, the subject was placed in his wheelchair on top of a $19 \mathrm{~cm}$ wooden platform. The object's height was adjusted using a mechanical jack (Figures $1 \mathrm{~b}$ and $\mathrm{c}$ ).

The evaluation focused on trunk movement. The grasp movement was not significant to the study but was included to imitate a daily activity.

Individuals with paraplegia performed the test in their personal wheelchairs to simulate their performance during activities of daily living and assess their dynamic postural control.

In addition, according to the literature, individuals with SCI have a posterior displaced center of mass when sitting. Most people with SCI sit with a slump and a posterior pelvic tilt of at least $15^{\circ} .{ }^{20,21}$ With respect to this, the participants were oriented to maintain the most upright position in the chair, avoiding pelvic retroversion. The control group sat in an armless chair and performed the same tests.

To avoid muscle fatigue, a 3- min rest was administered between each trial. The reach and grasp tasks were performed three times, and the mean rating was recorded for data analysis.

During the anterior and right lateral reach and grasp tests, EMG signals were recorded on the following muscles: longissimus, iliocostalis lumborum and multifidus, bilaterally.

The EMG signals were recorded using an eight-channel biological signal acquisition module (Delsys, Boston, MA, USA). The sample frequency was set to $4000 \mathrm{~Hz}$ with a total gain of 1000 times.

Active differential bars and bipolar $\mathrm{Ag} / \mathrm{AgCl}$ electrodes (Delsys) measuring $10 \times 1 \mathrm{~mm}$ with an inter electrode distance of $10 \mathrm{~mm}$ were positioned on the following muscles-iliocostalis lumborum, longissimus and multifidusaccording to Surface Electromyography for Non-Invasive Assessment of Muscles (SENIAM) recommendations (http://www.seniam.org/). The skin was prepared in accordance with the standardization of SENIAM to reduce skin impedance (http://www.seniam.org/). ${ }^{22}$

The EMG signals were processed in specific routines developed in Matlab (Mathworks Inc., Natick, MA, USA). A band-pass filter with cutoff frequency of $20-500 \mathrm{~Hz}$ was used to process the signals. The signals were full-wave

Table 1 Mean values \pm s.d. of volunteers' characterization

\begin{tabular}{|c|c|c|c|c|}
\hline & PASCI & $\mathrm{SSCl}$ & C & P-value \\
\hline Age (years) & $32.5( \pm 5.4)$ & $39.5( \pm 12.0)$ & $33.4( \pm 9.1)$ & 0.24 \\
\hline Weight (kg) & $71.8( \pm 13.5)$ & $78.1( \pm 13.7)$ & $77.5( \pm 8.8)$ & 0.45 \\
\hline Height (cm) & $177.3( \pm 7.2)$ & $174.1( \pm 8.4)$ & $174.6( \pm 5.0)$ & 0.55 \\
\hline BMI (kg m-2) & $22.9( \pm 4.1)$ & $25.7( \pm 3.3)$ & $25.4( \pm 2.9)$ & 0.15 \\
\hline Lesion time (years) & $11.8( \pm 8.2)$ & $10.8( \pm 8.6)$ & - & 0.40 \\
\hline Lesion level & $\mathrm{T} 3 \mathrm{~A}-\mathrm{T} 10 \mathrm{~A}$ & T4A-T9A & - & - \\
\hline Physically active time (years) & $4.5( \pm 4.67)$ & - & - & - \\
\hline
\end{tabular}

Abbreviations: BMI, body mass index $\left(\mathrm{kg} \mathrm{m}^{-2}\right)$; C, Control group; PASCI, Physically Active Spinal Cord Injured group; SSCI, Sedentary Spinal Cord Injured group. rectified and smoothed with a lowpass fourth-order Butterworth filter, creating a linear envelope with a cutoff frequency of $6 \mathrm{~Hz}$.

The mean of the linear envelope for each muscle group was organized in separate graphs, considering activation vs $\%$ time of task (every $5 \%$ of total time), to perform the activation analysis. In addition, to compare the muscle activation between groups, we considered the mean of the linear envelop during the task.

\section{Statistical analysis}

The sample size was set at 30 individuals (effect size: 3.77, sample power: 0.99 and $\alpha=0.05$ ), taking into consideration the mean and standard deviation of longissimus activation in AR75. The calculation was performed using GPower 3.1.

The mean and standard deviations were considered to characterize the sample, and the Student's $t$-test was used to compare the time of injury. One-way analysis of variance was used to determine differences between groups regarding covariants such as age, weight, height and body mass index, with a separate test being performed for each covariant using group as a factor.

Multivariate analysis of variance was performed to determine possible differences between groups regarding muscle activation using group (PASCI, SSCI and C) as the factor and the values of EMG activation obtained as a function of time of task execution (at each 5\% point of total time) as dependent variables. One-way multivariate analysis of variance was performed to analyze each reach and grip task (AR75, AR90, RLR75, RLR90), considering all times of execution of each respective task.

After the multivariate analysis of variance tests, multiple univariate analysis of variance followed by the post hoc Bonferroni test was performed to reveal a significant difference between groups. ${ }^{23}$ These univariate analysis of variance were performed using group as a factor and each time of execution as the dependent variable. We utilized the Bonferroni method to correct the $P$-value in the post hoc tests of the multivariate analysis of variance, thereby controlling the familywise error rate.

Mean activation per activity was considered for intragroup muscle comparison. The Hotelling T2 multivariate comparison test was performed to detect which muscle was most activated in each task in each group.

All statistical tests were applied after testing univariate and multivariate normality and after confirming variance homogeneity using the Shapiro-Wilk test for univariate normality, the Doornik-Hansen test for multivariate normality and the Batlett test for homogeneity. When necessary, the variables were transformed in order to satisfy these assumptions.

Data were analyzed statistically using the Statistics Data Analysis software-STATA 12.0 Special Edition-with the level of significance set at $\alpha=0.05$ in all analyses.

\section{RESULTS}

There were no significant differences between groups regarding age, weight, height, body mass index or time of injury. The sample characterization is presented in Table 1.

Data for 20 individuals with a complete traumatic SCI level between T3 and T10 on the American Spinal Injury Association scale were considered for the present study. ${ }^{17}$

Intragroup analysis of EMG activation during anterior reach and right lateral reach activities was performed to compare muscle activation during each task for each group (Table 2).

Comparison of the activities in the control group showed a highest mean activation of the left longissimus muscle during all activities (AR75: $P<0.00$; AR90: $P<0.00$; RLR75: $P=0.01$; RLR90, $P=0.02$ ) (Table 2A). In the PASCI group, a significantly highest activation of the left iliocostalis muscles was detected during all activities, except in the anterior reach task with $90 \%$ maximum reach (AR75: $P=0.02$; RLR75: $P=0.03$; RLR90: $P=0.01$ ) (Table 2B). In the SSCI group, a highest activation of the left iliocostalis was detected during the right lateral reach task of 75 and $90 \%$ maximum reach and of the right 
Table 2 Intragroup analysis of electromyographic activation of longissimus muscles, iliocostalis and multifidus muscles, bilaterally, during anterior reach and right lateral reach activities

\begin{tabular}{|c|c|c|c|c|c|c|c|}
\hline & $\operatorname{LBR}(\mu V)$ & $L B L(\mu V)$ & $I C R(\mu V)$ & $I C L(\mu V)$ & $M R(\mu V)$ & $M L(\mu V)$ & ap-value \\
\hline \multicolumn{8}{|l|}{ (A) Control group } \\
\hline AR Activities 75 & $14.12( \pm 7.22)$ & $15.56^{\mathrm{a}}( \pm 6.84)$ & $4.41( \pm 2.06)$ & $5.31( \pm 2.92)$ & $8.81( \pm 4.51)$ & $8.89( \pm 4.19)$ & $<0.001$ \\
\hline AR Activities 90 & $17.54( \pm 8.48)$ & $19.64^{\mathrm{a}}( \pm 8.19)$ & $5.25( \pm 2.90)$ & $6.20( \pm 4.00)$ & $10.16( \pm 4.75)$ & $10.31( \pm 4.32)$ & $<0.001$ \\
\hline RLR Activities 75 & $4.90( \pm 3.74)$ & $9.10^{\mathrm{a}}( \pm 4.16)$ & $3.04( \pm 2.24)$ & $4.98( \pm 2.22)$ & $3.65( \pm 2.70)$ & $5.88( \pm 2.81)$ & 0.01 \\
\hline RLR Activities 90 & $5.97( \pm 5.88)$ & $10.69^{a}( \pm 7.63)$ & $3.80( \pm 2.48)$ & $5.51(2.84)$ & $4.17( \pm 3.42)$ & $6.43( \pm 3.45)$ & 0.02 \\
\hline \multicolumn{8}{|l|}{ (B) PASCI group } \\
\hline AR Activities 75 & $6.67( \pm 4.34)$ & $7.46( \pm 4.27)$ & $7.27( \pm 5.60)$ & $12.68^{a}( \pm 12.35)$ & $3.92( \pm 2.08)$ & $6.30( \pm 7.44)$ & 0.02 \\
\hline AR Activities 90 & $7.39( \pm 4.87)$ & $8.22( \pm 5.03)$ & $6.71( \pm 4.23)$ & $10.74( \pm 5.43)$ & $4.27( \pm 2.48)$ & $7.04( \pm 8.85)$ & - \\
\hline RLR Activities 75 & $4.78( \pm 1.95)$ & $8.03( \pm 7.40)$ & $4.79( \pm 2.52)$ & $16.29^{a}( \pm 10.22)$ & $2.78( \pm 0.75)$ & $3.12( \pm 0.93)$ & 0.03 \\
\hline RLR Activities 90 & $4.57( \pm 1.83)$ & $7.49( \pm 5.00)$ & $5.46( \pm 3.50)$ & $17.92^{\mathrm{a}}( \pm 12.18)$ & $2.88( \pm 0.72)$ & $6.60( \pm 10.60)$ & 0.01 \\
\hline \multicolumn{8}{|l|}{ (C) SSCl group } \\
\hline AR Activities 75 & $6.88( \pm 4.55)$ & $5.26( \pm 3.03)$ & $11.85^{\mathrm{a}}( \pm 12.83)$ & $9.65( \pm 8.81)$ & $3.02( \pm 1.44)$ & $2.65( \pm 1.21)$ & 0.007 \\
\hline AR Activities 90 & $7.72( \pm 5.21)$ & $6.76( \pm 7.04)$ & $13.36( \pm 14.41)$ & $11.26( \pm 8.55)$ & $3.68( \pm 2.04)$ & $2.73( \pm 1.09)$ & - \\
\hline RLR Activities 75 & $4.12( \pm 2.53)$ & $4.68( \pm 3.37)$ & $5.86( \pm 6.92)$ & $10.82^{\mathrm{a}}( \pm 8.15)$ & $2.58( \pm 0.95)$ & $2.80( \pm 1.90)$ & 0.02 \\
\hline RLR Activities 90 & $3.90( \pm 2.35)$ & $5.00( \pm 3.88)$ & $5.84( \pm 5.98)$ & $12.11^{a}( \pm 10.46)$ & $2.58( \pm 0.95)$ & $2.75( \pm 1.62)$ & 0.03 \\
\hline
\end{tabular}

Abbreviations: RLR activities 75 and $90=$ right lateral reach activities to $75 \%$ and $90 \%$ of maximum reach; C, Control group; ICR and ICL, iliocostalis muscles right and left; LBR and LBL, longissimus muscles of the back right and left; MR and ML, multifidus muscles right and left; PASCI, Physically Active Spinal Cord Injured group; SSCI, Sedentary Spinal Cord Injured group. Values presented as the means \pm s.d.

${ }^{a}$ Muscles that presented higher activation during the task $(P<0.05)$.

iliocostalis during the anterior reach task of $75 \%$ maximum reach (AR75: $P=0.00$; RLR75: $P=0.02$; RLR90: $P=0.03$ ) (Table 2C).

Regarding the time needed to perform the activities, we detected no significant differences in the mean data among the groups during the reaching tasks.

The group comparisons (C, PASCI and SSCI) regarding mean values obtained through the three trials of muscle activation during time points in each activity are presented in Figures 2-5.

\section{Anterior reach activities of $75 \%$ maximum reach}

Regarding anterior reach activity of $75 \%$ maximum reach, we observed greater activation of the right and left logissimus muscles in the control group compared with the SCI groups at most time points during activity (12 and 17 time points, respectively, in the two groups). During this activity, we observed a greater activation of the right multifidus muscle in the control group compared with the SCI group at most time points (12 time points compared with the physically active SCI group and 13 time points compared with the sedentary SCI group) (Figure 2).

A greater activation of the left multifidus muscle was observed in the control group compared with the SCI groups at some time points during this activity (6 time points for both groups).

\section{Anterior reach activities of $\mathbf{9 0 \%}$ maximum reach}

Regarding anterior reach activity of $90 \%$ maximum reach, we observed greater activation of the right longissimus muscle in the control group compared with the SCI groups at most time points during the activity (15 time points in both groups) (Figure 3 ).

A greater activation of the left longissimus muscle was observed in the control group compared with the SCI groups at most time points during this activity ( 15 time points compared with the physically active SCI group and 16 time points compared with the sedentary SCI group).

A greater activation of the left iliocostal muscle was observed in the control group compared with the active SCI group only at 1 time point during this activity.
A greater activation of the right multifidus muscle was observed in the control group compared with the SCI groups at most time points during this activity (17 time points compared with the physically active SCI group and 18 time points compared with the sedentary SCI group).

A greater activation of the left multifidus muscle was observed in the control group compared with the SCI groups at some time points during this activity (6 time points in both groups), as well as in the physically active SCI group compared with the sedentary SCI group (2 time points).

Right lateral reach activities of $75 \%$ maximum reach

Regarding right lateral reach activity of $75 \%$ maximum reach, a greater activation of the left longissimus muscle was observed in the control group compared with the SCI groups at some time points during the activity (8 time points in both groups) (Figure 4).

A greater activation of the left iliocostal muscle was observed in the physically active SCI group compared with the control and sedentary SCI groups ( 9 time points compared with control and 5 time points compared with the sedentary SCI group).

A greater activation of the left multifidus muscle was observed in the control group compared with the SCI groups at some time points during this activity ( 8 time points in both groups).

\section{Right lateral reach activities of $\mathbf{9 0 \%}$ maximum reach}

During the right lateral reach activity of $90 \%$ maximum reach, a greater activation of the left longissimus muscle was observed in the control group compared with the SCI groups at some time points during the activity (9 time points in both groups), as well as in the physically active SCI group compared with control and with the sedentary SCI group ( 2 time points compared with control and 3 time points compared with the sedentary SCI group) (Figure 5).

A greater activation of the left iliocostal muscle was observed in the physically active SCI group compared with control and with the sedentary SCI group during this activity ( 8 time points compared with control and 4 time points compared with the sedentary SCI group), 

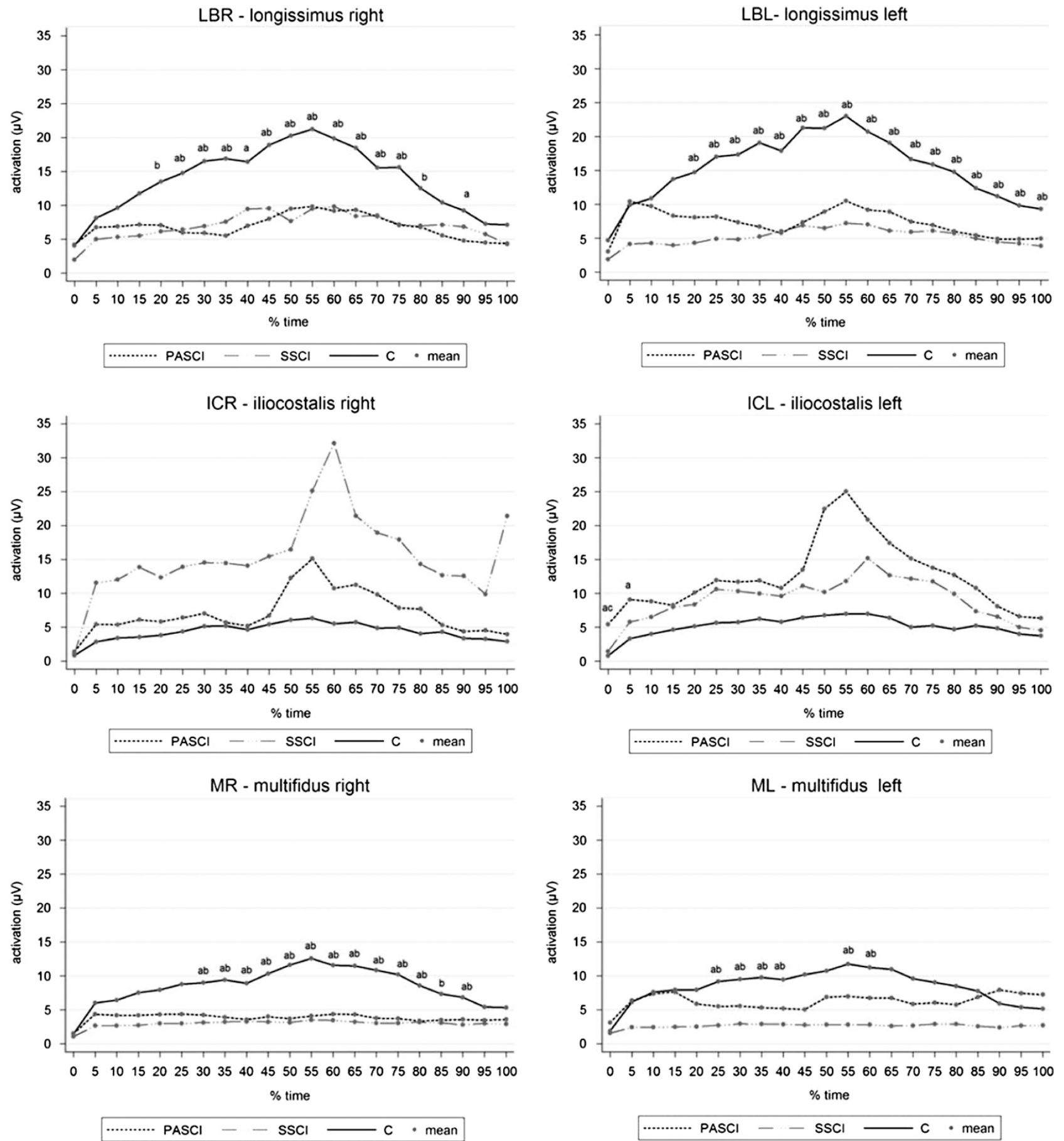

Figure 2 Intergroup analysis of electromyographic activation of longissimus muscles, iliocostalis and multifidus muscles, bilaterally, during anterior reach activities of $75 \%$ maximum reach considering activation vs $\%$ time of task. Mean values of three trials are presented for each time point. $a=P<0.05 \mathrm{C}$ vs PASCl; $\mathrm{b}=P<0.05 \mathrm{C}$ vs SSCl. C, Control group; PASCI, Physically Active Spinal Cord Injured group; SSCI, Sedentary Spinal Cord Injured group.

as well as a greater muscle activation in the sedentary SCI group compared with control at 3 time points during this activity.

A greater activation of the left multifidus muscle was observed in the control group compared with the sedentary SCI group at 2 time points.

\section{DISCUSSION}

Individuals with SCI may use abnormal trunk movement compensate for motor control dysfunction and instability. ${ }^{9,24}$ There are no studies that investigate trunk activation in active and sedentary individuals with paraplegia. This study was conducted to identify whether adapted sports altered trunk muscle activation and the impact of sports practice on postural control.

The iliocostalis, multifidus and longissimus are muscles responsible for movements such as trunk extension and trunk lateral flexion. In addition, the iliocostalis and longissimus muscles pull the ribs down. ${ }^{25}$

These dorsal muscles of the trunk, that is, the iliocostalis, multifidus and longissimus muscles, also have a stabilizing function, which is 

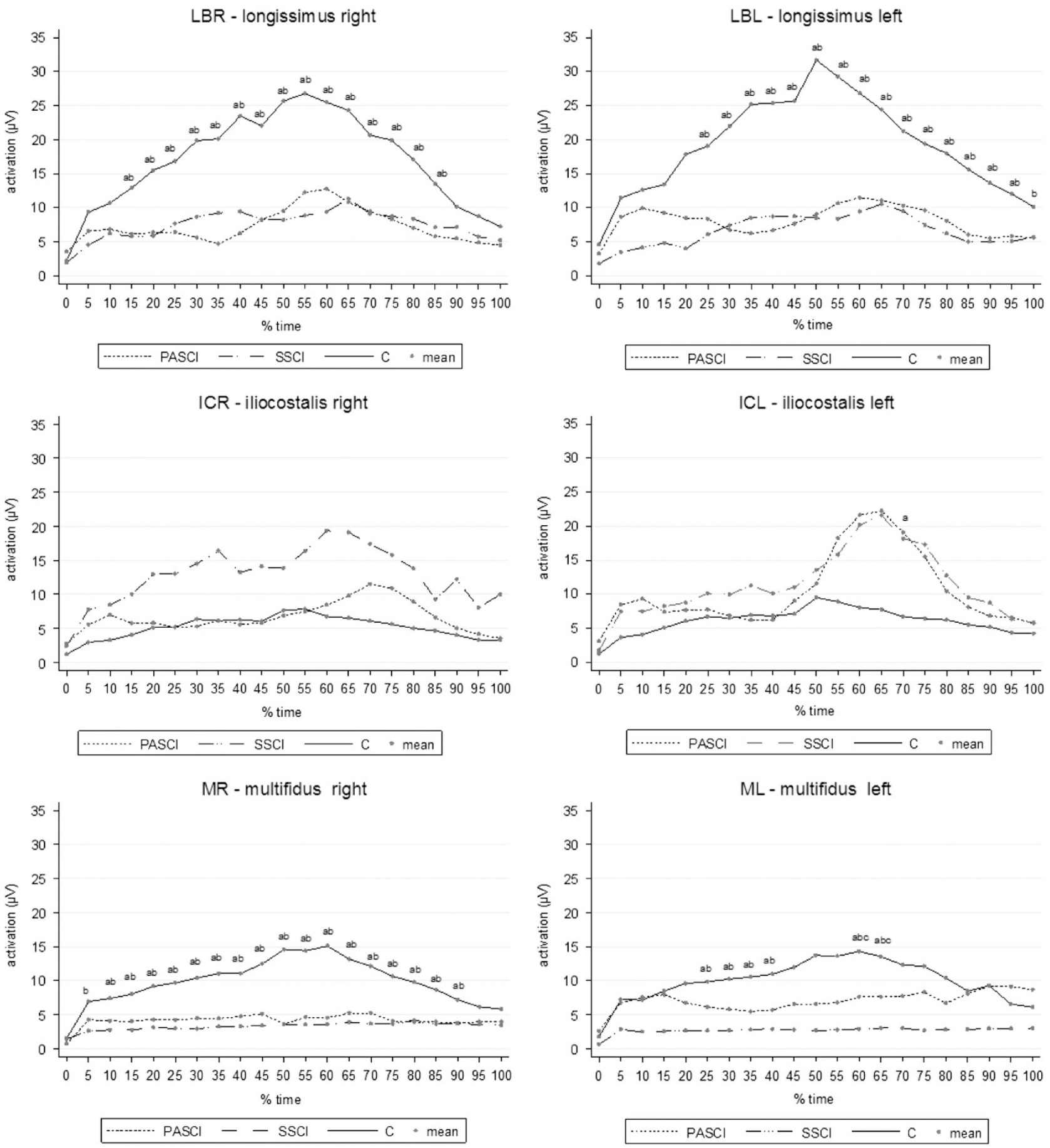

Figure 3 Intergroup analysis of electromyographic activation of longissimus muscles, iliocostalis and multifidus muscles, bilaterally, during anterior reach activities of $90 \%$ maximum reach considering activation vs $\%$ time of task. Mean values of three trials are presented for each time point. $a=P<0.05 \mathrm{C}$ vs PASCl; $\mathrm{b}=P<0.05 \mathrm{C}$ vs SSCl; $\mathrm{c}=P<0.05 \mathrm{PASCl}$ vs SSCl. C, Control group; PASCl, Physically Active Spinal Cord Injured group; SSCl, Sedentary Spinal Cord Injured group.

required to perform a movement without losing the balance. No trunk muscle contributed more than $30 \%$ to lumbar spine stabilization. Thus, lumbar spine stability depends on the general activation of trunk muscles. ${ }^{26}$

Individuals with SCI do not have good control of stabilizing muscles of the spine. Probably for this reason, our results demonstrated a different activation pattern when we compared the control group with the SCI group. ${ }^{10}$
Unlike the control group, both SCI groups had a significantly highest activation of the left iliocostalis muscle during the anterior reach task and right lateral reach task. Because this activation was only found in subjects with paraplegia it could represent postural compensation in order to maintain stability during these movements.

Thus, as people with SCI do not have efficient control of muscle activation below the injury level, they may learn a new motor control 

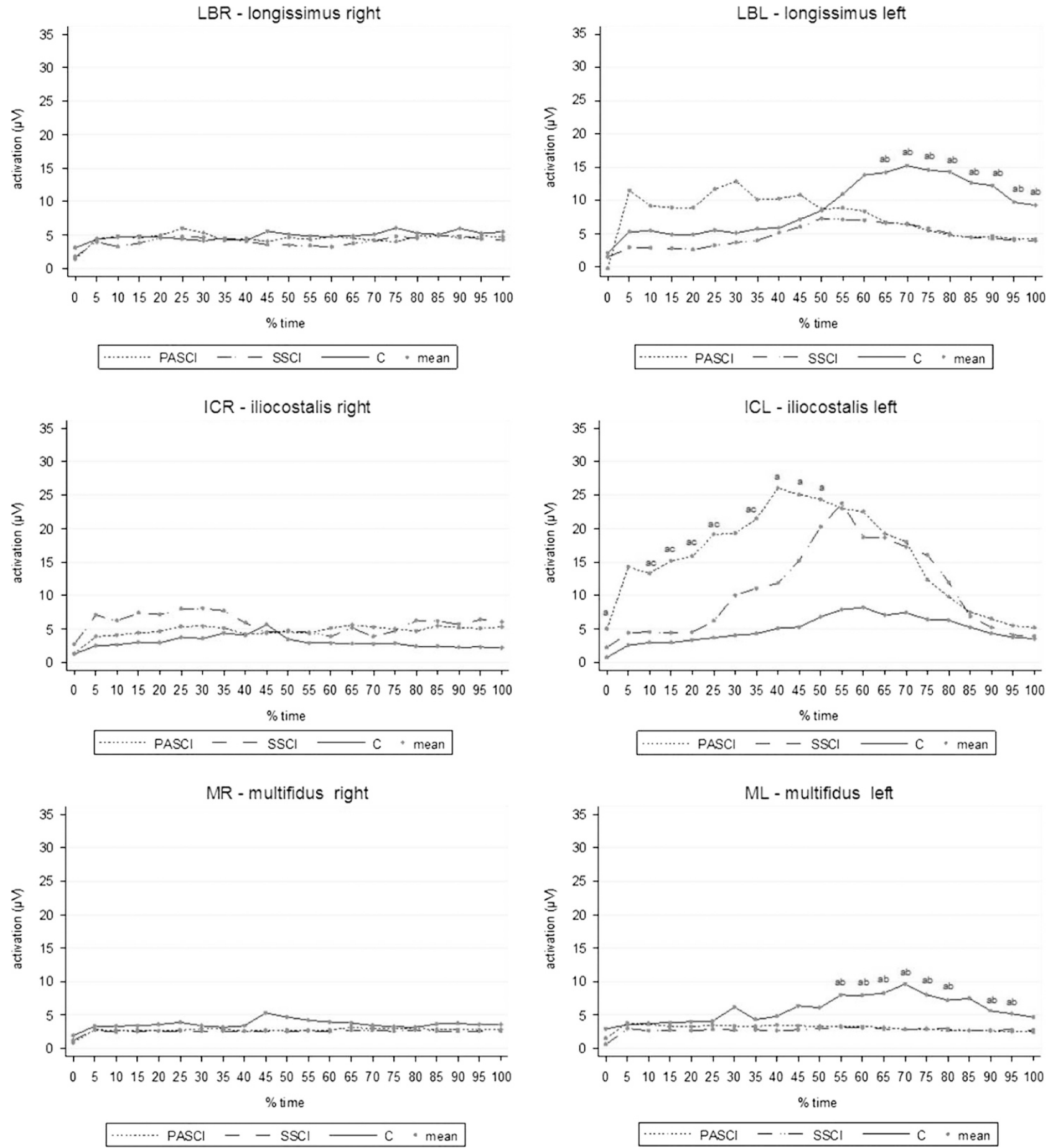

Figure 4 Intergroup analysis of electromyographic activation of longissimus muscles, iliocostalis and multifidus muscles, bilaterally, during right lateral reach activities of $75 \%$ maximum reach considering activation vs $\%$ time of task. Mean values of three trials are presented for each time point. $a=P<0.05 \mathrm{C}$ vs PASCl; $\mathrm{b}=P<0.05 \mathrm{C}$ vs SSCl; $\mathrm{c}=P<0.05 \mathrm{PASCl}$ vs SSCI. C, Control group; PASCI, Physically Active Spinal Cord Injured group; SSCI, Sedentary Spinal Cord Injured group.

pattern to maintain stability. The new motor control pattern might recruit muscles with intact innervation, altering the recruitment of trunk muscles, as identified in the present study.

In a case study, Bjerkefors (2009) investigated the activation of trunk muscles (abdominal muscles, erector spinae and muscles of the upper trunk) below the level of spine injury during tasks such as flexion, extension and inclination with external disturbances in subjects who had complete T3 injury. The author observed that the subject with paraplegia was able to activate all of the muscles below the injury level. However, the author found a difference in the time and amount of muscle activation between a subject with SCI and an individual without SCI. ${ }^{18}$ Thus, our results support the findings of Bjerkefors et al. ${ }^{27}$ because we observed muscle activation below the level of injury in individuals with SCI. 

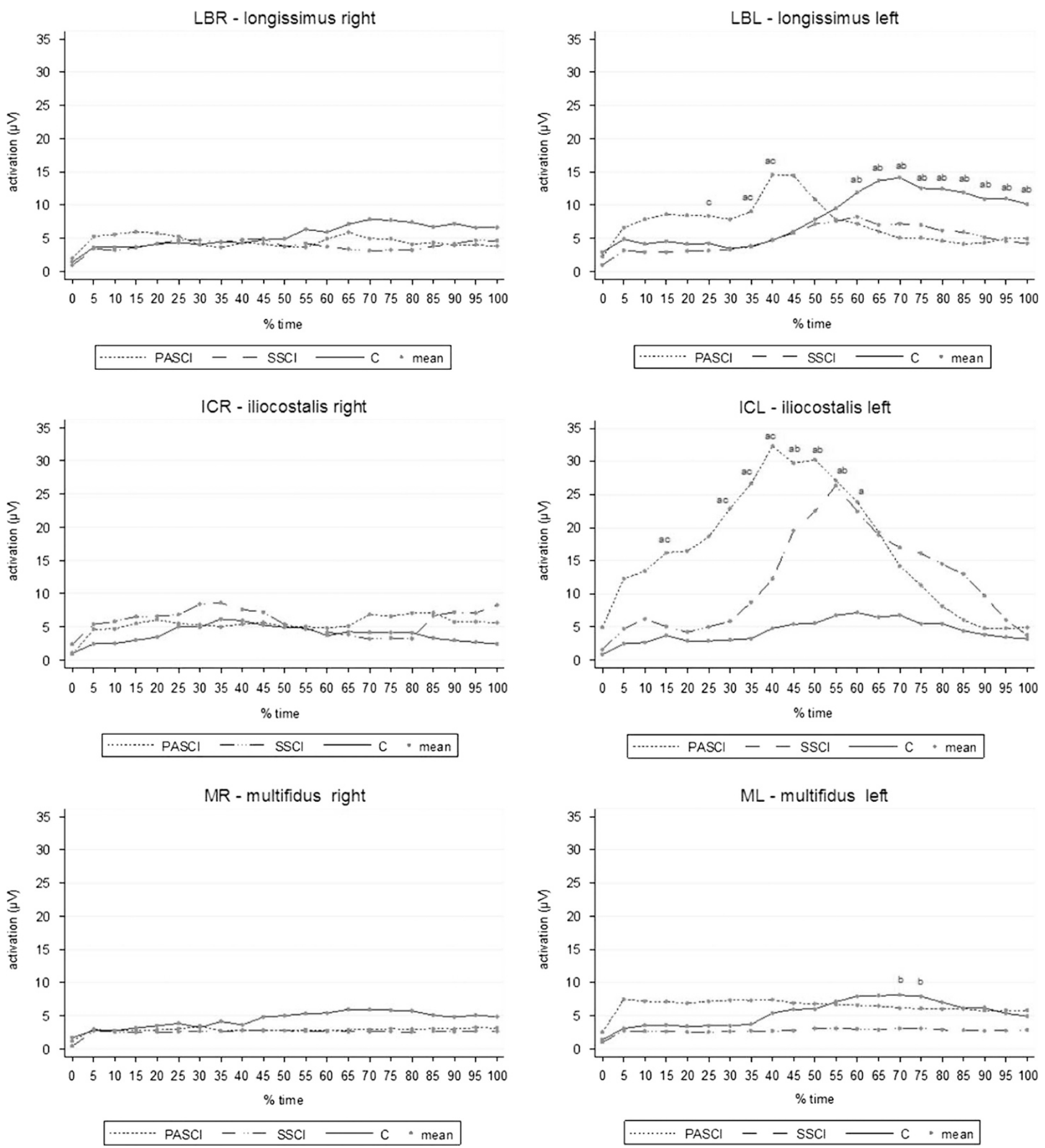

Figure 5 Intergroup analysis of electromyographic activation of longissimus muscles, iliocostalis and multifidus muscles, bilaterally, during right lateral reach activities of $90 \%$ maximum reach considering activation vs \% time of task. Mean values of three trials are presented for each time point. $a=P<0.05 \mathrm{C}$ vs $\mathrm{PASCl} ; \mathrm{b}=P<0.05 \mathrm{C}$ vs SSCI; $\mathrm{c}=P<0.05 \mathrm{PASCl}$ vs SSCI. C, Control group; PASCl, Physically Active Spinal Cord Injured group; SSCl, Sedentary Spinal Cord Injured group.

In the present study, we observed a different pattern of muscle activation between the control group and the SCI groups. During anterior activities of 75 and $90 \%$ maximum reach, a greater activation of the longissimus muscles of the dorsum and of the right multifidus in the control group compared with the SCI group at most time points was observed. However, we cannot state that we detected a different pattern of activation between groups regarding other muscles or other activities, as differences were observed in less than $50 \%$ of the time points.

We did not detect any difference between groups regarding the pattern of trunk muscle activation in the lumbar region during lateral reach, probably because this activity requires most activation of thoracic rather than lumbar muscles, causing a very small movement amplitude and muscle activation in this region. ${ }^{28}$ 
The practice of many sports requires agility, such as throwing the ball quickly, and these movements can be performed with different patterns, as with the arm only or with arm and trunk movements. When the athlete uses arm and trunk movements, this alters their center of mass and requires compensatory responses to maintain stability in the sitting position. ${ }^{29}$ This study agrees with our hypothesis that adapted sports can alter trunk muscle activation, but we cannot confirm this initial hypothesis.

A recent study observed that subjects with SCI who practice wheelchair sports are able to change the direction of their trunk (anteroposterior and laterolateral) more rapidly compared with sedentary subjects with SCI, and the laterolateral movement was the one most rapidly performed, suggesting that physical activity can improve laterolateral postural control. In the present study, we detected no difference between groups regarding the time needed to perform anterior and lateral reach activities. ${ }^{30}$

Seleen and colleagues ${ }^{24}$ identified that a different muscle activation pattern may occur in order to compensate the functional deficit and trunk instability due to SCI dysfunction. Our results show that individuals with SCI use different lumbar muscle activation strategies compared with individuals without injury to accomplish these tasks.

Our results show that the sport did not interfere with trunk muscle activation during anterior and lateral reach and grasp activities. These results contradict the hypothesis of our study.

For future investigations, we suggest identifying strategies used by individuals with SCI to perform these activities. The investigation can be helpful in specifically guiding and training these muscles to improve the postural control and balance of athletes with paraplegia.

The limitations of our study included the reduced muscle groups assessed, the absence of kinematic analysis to observe the relation between electromyography and kinematic performance and the lack of use of surface markers and other potentials for cross-talk. Also, EMG signals were not normalized by maximal voluntary contraction or other techniques. Thus, some factors such as fat tissue may impact the comparison between groups and extrapolation should be made with caution.

We conclude that sports practice did not affect the trunk muscle activation in people with paraplegia. However, the pattern muscle activation in individuals with SCI is different compared with people without SCI during anterior reach tasks.

\section{DATA ARCHIVING}

There were no data to deposit.

\section{CONFLICT OF INTEREST}

The authors declare no conflict of interest.

\section{ACKNOWLEDGEMENTS}

We thank the São Paulo Research Support Foundation (FAPESP—2013/15547-5) for providing financial support.
1 Horak FB. Postural orientation and equilibrium: what do we need to know about neural control of balance to prevent falls? Age Ageing 2006; 35(Suppl: 2): ii7-ii11.

2 Pollock AS, Durward BR, Rowe PJ, Paul JP. What is balance? Clin Rehabil 2000; 14: 402-406.

3 Shumway-Cook A, Woollacott MH. Motor Control: Translating Researh into Cinical Pratice, 3rd edn. Lippincott Williams Wilkins: Philadelphia, PA, USA, 2003, pp 3-299.

4 Liechti M, Muller R, Lam T, Curt A. Vestibulospinal responses in motor incomplete spinal cord injury. Clin Neurophysiol 2008; 119: 2804-2812.

5 Bradbury EJ, McMahon SB. Spinal cord repair strategies: why do they work? Nat Rev Neurosci 2006; 7: 644-653.

6 Seelen HA, Potten YJ, Huson A, Spaans F, Reulen JP. Impaired balance control in paraplegic subjects. J Electromyogr Kinesiol 1997; 7: 149-160.

7 Seelen HA, Janssen-Potten YJ, Adam JJ. Motor preparation in postural control in seated spinal cord injured people. Ergonomics 2001; 44: 457-472.

8 Seelen HA, Potten YJ, Adam JJ, Drukker J, Spaans F, Huson A. Postural motor programming in paraplegic patients during rehabilitation. Ergonomics 1998; 41: 302-316.

9 Chen $\mathrm{CL}$, Yeung KT, Bih LI, Wang $\mathrm{CH}$, Chen MI, Chien JC. The relationship between sitting stability and functional performance in patients with paraplegia. Arch Phys Med Rehabil 2003; 84: 1276-1281.

10 Reft J, Hasan Z. Trajectories of target reaching arm movements in individuals with spinal cord injury: effect of external trunk support. Spinal Cord 2002; 40: 186-191.

11 Atrice MB, Morrison SA, McDowell SL, Shandalov B. Spinal cord injury. In: Umphred DA (ed.). Neurological Rehabilitation, 4 edn. Barueri: St Louis, MO, USA, 2004.

12 Hassall CD, MacLean S, Krigolson OE. Hierarchical error evaluation: the role of medial-frontal cortex in postural control. J Mot Behav 2014; 46: 381-387.

13 Moreno MA, Zamune'r AR, Paris JV, Teodori RM, Barros RML. Effects of wheelchair sports on respiratory muscle strength and thoracic mobility of individuals with spinal cord injury. Am J Phys Med Rehabil 2012; 91: 470-477.

14 Manns PJ, Chad KE. Determining the relation between quality of life, handicap, fitness, and physical activity for persons with spinal cord injury. Arch Phys Med Rehabil 1999; 80: 1566-1571.

15 De Luca CJ. The use of surface electromyography in biomechanics. J Appl Biomech 1997; 13: 135-163.

16 Sprigle S, Wootten M, Sawacha Z, Thielman G. Relationships among cushion type, backrest height, seated posture, and reach of wheelchair users with spinal cord injury. J Spinal Cord Med 2003; 26: 236-243.

17 El Masry WS, Tsubo M, Katoh S, El Miligui YH, Khan A. Validation of the American Spinal Injury Association (ASIA) motor score and the National Acute Spinal Cord Injury Study (NASCIS) motor score. Spine (Phila Pa 1976) 1996; 21: 614-619.

18 Duncan PW, Weiner DK, Chandler J, Studenski S. Functional reach: a new clinical measure of balance. J Gerontol 1990; 45: M192-M197.

19 Lynch SM, Leahy P, Barker SP. Reliability of measurements obtained with a modified functional reach test in subjects with spinal cord injury. Phys Ther 1998; 78: 128-133.

20 Hobson DA. Comparative effects of posture on pressure and shear at the body-seat interface. J Rehabil Res Dev 1992; 29: 21-31.

21 Hobson DA, Tooms RE. Seated lumbar/pelvic alignment: a comparison between spinalcord injured and noninjured groups. Spine 1992; 17: 293-298.

22 Hermens HJ, Freriks B, Disselhorst-Klug C, Rau G. Development of recommendations for SEMG sensors and sensor placement procedures. J Electromyogr Kinesio/ 2000; 10: 361-374.

23 Hair JF Jr, Black WC, Babin BJ, Anderson RE, Multivariate Data Analysis, 7th edn. Prentice Hall: Pearson Education Limited, Upper Saddle River, 2009.

24 Seelen HA, Potten YJ, Drukker J, Reulen JP, Pons C. Development of new muscle synergies in postural control in spinal cord injured subjects. J Electromyogr Kinesiol 1998; 8: 23-34.

25 Kendall FP, McCreary EK, Provance PG. MMR Músculos: Provas e Funções, 5th edn. Manole, 2007, pp 165-244.

26 Willson JD, Dougherty CP, Ireland ML, Davis IM. Core stability and its relationship to lower extremity function and injury. J Am Acad Orthopaed Surg 2005; 13: 316-325.

27 Bjerkefors A, Carpenter MG, Cresswell AG, Thorstensson A. Trunk muscle activation in a person with clinically complete thoracic spinal cord injury. J Rehabil Med 2009; 41: 390-392.

28 White AA, Panjabi MM. Clinical Biomechanics of the Spine, 2nd edn. Lippincott: Philadelphia, 1990.

29 Janssen-Potten YJ, Seelen HA, Drukker J, Reulen JP. Chair configuration and balance control in persons with spinal cord injury. Arch Phys Med Rehabil 2000; 81: 401-408.

30 Santos SS, Monteiro CBM, Cantelli B, Alonso AC, Mochizuki L, Nicolai Ré AH et al. Analysis of velocity and direction of trunk movement in wheelchair basketball athletes. Medical Express 2014; 1: 77-80. 